\title{
Mental Training in Sports: Summary and Perspectives
}

\author{
Malkin Valeriy ${ }^{1}$, Rogaleva Lyudmila ${ }^{1}$, Kim Alla $^{2}$, Aidossova Zhanerke ${ }^{2}$, Khon Natalya ${ }^{3}$ \\ ${ }^{1}$ The Ural federal University named after the first President of Russia B.N. El'tsin \\ ${ }^{2}$ al-Farabi Kazakh National University Almaty, Kazakhstan \\ ${ }^{3}$ Russian - Armenian (Slavonic) University, Republic of Armenia \\ valerymalkin@yandex.ru, liudmila.rogaleva@yandex.ru, a.kim@inbox.ru, zhanerke90@mail.ru, natalya.khon@gmail.com
}

\begin{abstract}
Psychological stability of an athlete is an important factor of achievement high results in sports. To solve this problem the mental training has been used. Its weakness consists of limitation of the regulation process by mechanisms of physiological level only. The perspectives for the development of mental training in sport will be dependent from the result of implementation of psychological mechanisms of motivational-emotional level of athletes. Methodology of psychoforming training based on the development of system of attitudes has been elaborated. The results revealed rather high effectiveness of the psychoforming training as a mean of stabilizing the psychic state of an athlete in competitions.
\end{abstract}

Index Terms - mental training, psychoforming training, attitudes change, self-confidence

\section{Introduction}

Mental training in sport has been used in sports for more than 60 years $[1,2,3,4,5,6]$.

A lot of research were organized for examining the impact of mental training on different functional systems of organism and psychological condition of sportsman during both the practice and competition. The positive influence of mental training on functioning the organism has been revealed. These data allow us to conclude about the role of mental training in sports and to draw some perspectives. It is important to stress the role of mental training in sports now because it is supposed to be underestimated despite of its usefulness. Our experience as psychologists of sports in national and professional teams of Russia for many years shows that even elite sportsmen do not use mental training in their preparation, with seldom exception. In children and youth sport the situation is even worse. The situation in other countries, Kazakhstan, for example is similar. Unfortunately there are serious reasons for appearance of such situation in using mental training in sport. First of all, mental training in its current state cannot guarantee constant success of sportsman in the most important competitions, although many psychologists promise such effect to the sportsmen.

\section{Method}

More than thirty years history of research of mental training as a factor forming psychological stability of a sportsman in competitions revealed that it supports physiological regulatory processes and provides optimal functioning of organism at vegetative and psychomotor level only. It can provide stability by exercise performance. However, physiological mechanisms, which could be trained by the mental training, can stabilize performance only till definite limit of stress. Beyond this limit the functional possibilities of organism are disorganized and successful performance is obstructed. Nevertheless, psychologists would like to transfer positive effect of physiological mechanisms to the psychological sphere of an athlete.

But these are psychological mechanisms (attitudes, motives, self-esteem, capability for voluntary regulation) which start to regulate psychological condition of the sportsman under stress-competition conditions where responsibility for the result of performance is very high. At the same time the role of vegetative mechanisms comes down as soon as mechanisms of psychological level start to play the leading role. So, stabilization of psychological condition of athletes cannot be supported by the mechanisms of physiological level only.

It explains why athletes and coaches are uninterested in implementation of mental training which seems not to be able to provide any constant success in important competitions. The perspectives for the development of mental training in sport will be dependent from the result of implementation of psychological mechanisms of motivational-emotional level of athletes.

To solve this problem specific psychoforming training [7] for the development of system of attitudes has been elaborated.

Theory of A.N. Uznadze [8] regarding regulation and stabilization role of attitudes, regulating mental life and behaviour could be theoretical base for such a training.

The development of systems of attitudes was conducted in four stages (directing, forming, realizing, stabilizing). The first one was aimed at forming directing attitude for realization of necessary preliminary programs. This stage includes relaxation and concentration exercises. Psychological background is concentration-calmness- self- confidence.

The second stage was aimed at forming systems of attitudes (motivational, emotional and purposeful) by the development of adequate self-esteem and realistic aspiration level. First of all motivated self-suggestion and imperative self-suggestion were used. The analysis of "what I want, can and need to do" for transforming "my wishes" into "my competences" was conducted. Visual presentation, selfpersuasion, self- control were means of formation of these attitudes. 
The third stage was aimed at realizing and securing the functioning of system of attitudes under the conditions of competition as well as forming the blocking mechanism against influence of different negative factors such as coach, rival, unfavourable situation. This is a factor making possible to expect positive effect from using psychoforming training for mental state stabilization of sportsman in important competition. By the development of motivational attitudes the specific kind of activity and psychological obstacles in this activity were taken into account. Attitudes for selfactualization of inner resources in the condition of competition as a motivational attitude for competition were used. Selfanalysis, self-persuasion, motivating self-suggestion were used. Emotional attitude "self-confidence", "self-confidence in any situations, under any conditions" becomes background for the development of any specific and conditional attitudes of self-assurance.

The fourth stage was aimed at formation of individual attitude programs of sportsman. In the course of their formation and actualization some emotional and motivational spheres of personality have been reorganized.

\section{Results}

The balance between motive of achievement of success and avoiding of failure $(16.5 \pm 2.5$ to $\mathrm{I} 3.5 \pm 1.3, \mathrm{p}<0.01$ and $9.4 \pm 1,3$ to $12,0+\mathrm{p}<0.01$ ) and reduction of personal alarm $(41.9 \pm 5.0$ to $31,5+1.4, \mathrm{p}<0.05)$ was registered.

\section{Conclusion}

These changes characterize the personality, which becomes more active, confident and emotionally stable. At the same time blocking of switching from the conscious level of regulation to the unconscious one as well as blocking of attitudes inadequate to the conditions of competition were observed.

The results revealed rather high effectiveness of the psychoforming training as a mean of stabilizing the psychic state of athletes in competition.

\section{References}

[1] Unestahl, L.-E. (2011). Hypnosis, self-hypnosis and mental training. Stockholm: Mental Training Sweden AB.

[2] Alekseev, A.V. (2003). To overcome yourself! Moscow: Physical culture and Sport.

[3] Giessen, L.D. (1990). Stress time. Moscow: Physical culture and Sport.

[4] Gould, D. (2006). Goal setting for peak performance. In J. M.Williams (Ed.). Applied sport psychology: Personal growth to peak performance (5th ed., pp. $240-259)$. Boston: McGraw-Hill.

[5] Williams, J. M. (Ed.). (2006). Applied sport psychology: Personal growth to peak performance (5th ed.). Boston: McGraw-Hill.

[6] Kim A. M., Khon N. N. (2015) Psychology of pre-start arousal in equestrial sport//Emotional Intelligence in coach and exercise instructor acivity. Almaty: KazNU. p. 138-143.

[7] Malkin, V. R. (2008). Management psychological preparation in sports. Moscow: Physical culture and Sport.

[8] Uznadze, A. N. (2001). Psychology of attitude. St.-Peterburg: Peter. 